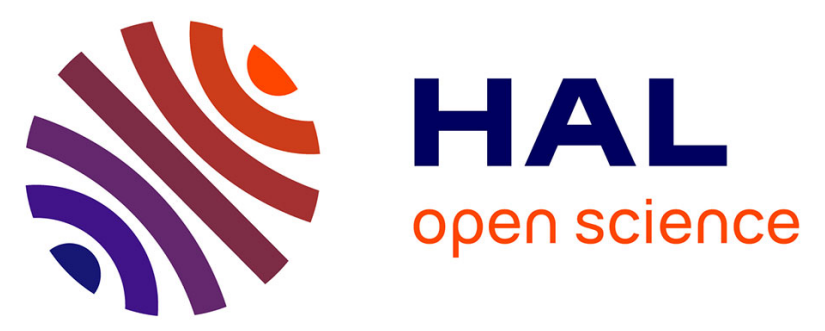

\title{
A novel dual-polarized continuous transverse stub antenna based on corrugated waveguides -Part I: principle of operation and design
}

Maciej Smierzchalski, Francesco Foglia Manzillo, Michele del Mastro, Nicolas Capet, Baptiste Palacin, Ronan Sauleau, Mauro Ettorre

\section{To cite this version:}

Maciej Smierzchalski, Francesco Foglia Manzillo, Michele del Mastro, Nicolas Capet, Baptiste Palacin, et al.. A novel dual-polarized continuous transverse stub antenna based on corrugated waveguides Part I: principle of operation and design. IEEE Transactions on Antennas and Propagation, 2021, 69 (3), pp.1302-1312. 10.1109/TAP.2020.3028236 . hal-03137417

HAL Id: hal-03137417 https://hal-univ-rennes1.archives-ouvertes.fr/hal-03137417

Submitted on 10 Feb 2021

HAL is a multi-disciplinary open access archive for the deposit and dissemination of scientific research documents, whether they are published or not. The documents may come from teaching and research institutions in France or abroad, or from public or private research centers.
L'archive ouverte pluridisciplinaire HAL, est destinée au dépôt et à la diffusion de documents scientifiques de niveau recherche, publiés ou non, émanant des établissements d'enseignement et de recherche français ou étrangers, des laboratoires publics ou privés. 


\title{
A Novel Dual-Polarized Continuous Transverse Stub Antenna Based on Corrugated Waveguides - Part I: Principle of Operation and Design
}

\author{
Maciej Śmierzchalski, Francesco Foglia Manzillo, Member, IEEE, Michele Del Mastro, Nicolas Capet, Baptiste \\ Palacin, Ronan Sauleau, Fellow, IEEE, and Mauro Ettorre, Senior Member, IEEE
}

\begin{abstract}
Continuous transverse stub (CTS) arrays represent a high-performance and affordable solution for fixed-beam and scanning flat antenna systems, though intrinsically linearlypolarized. In these systems, the polarization is controlled using add-on polarization converters or combining cross-connected CTS arrays. This two-part paper presents a novel parallelfed CTS antenna which achieves dual-linear polarization using, instead, a single array and two orthogonally polarized excitation fields. In this first Part, it is shown that, due to modal coupling, standard parallel-plate waveguides (PPWs) are not suitable as building blocks of the proposed antenna. Therefore, an alternative waveguide, the corrugated PPW (CPPW) is introduced. The dispersion characteristics of such CPPW are investigated. Guidelines to independently design the dispersion properties of the two propagating modes and avoid the excitation of higher order modes are provided. Based on this study, the design and simulated performance of a Ka-band array of eight long slots parallel-fed by a dual-mode CPPW corporate-feed network are described. An extensive experimental validation of the concept is presented in Part II.
\end{abstract}

Index Terms-dual-polarization antennas, high-gain antennas, satellite communications, Ka-band, aperture antennas

\section{INTRODUCTION}

$\mathbf{M}$ ODERN satellite communication (Satcom) systems have been rapidly evolving in last years. They are currently designed to operate at higher frequencies, often at Ka-band, to increase the throughput and use circular polarization to avoid depolarizing effects. Ground terminals require scanning antennas to seamlessly track satellites, especially for communications on-the-move. Light weight and low profile

This work has been funded in part by the Centre National d'Etudes Spatiales (CNES), in part by the European Union through the European Regional Development Fund and in part by the French Region of Brittany, Ministry of Higher Education and Research, Rennes Métropole and Conseil Départemental 35 through the CPER Project STIC \& Ondes.

M. Śmierzchalski was with Univ Rennes, CNRS, IETR (Institut d'Electronique et de Télécommunications de Rennes), UMR 6164, F35000 Rennes. He is now with Atos, 95877 Bezons, and also with Univ. Grenoble Alpes, CEA, Leti, 38000 Grenoble, France (e-mail: maciej.smierzchalski@cea.fr).

F. Foglia Manzillo was with Univ Rennes, CNRS, IETR (Institut d'Electronique et de Télécommunications de Rennes), UMR 6164, F-35000 Rennes. He is now with Univ. Grenoble Alpes, CEA, Leti, 38000 Grenoble, France (e-mail: francesco.fogliamanzillo@cea.fr).

M. Del Mastro, R. Sauleau and M. Ettorre are with Univ Rennes, CNRS, IETR (Institut d'Electronique et de Télécommunications de Rennes), UMR 6164, F-35000 Rennes (e-mails: \{michele.delmastro, ronan.sauleau, mauro.ettorre\}@univ-rennes1.fr).

N. Capet and B. Palacin are with the Antenna Department, Centre National d'Etudes Spatiales (CNES), Toulouse 31401, France (e-mails: nicolas.capet@anywaves.fr; baptiste.palacin@cnes.fr). are requested to fulfill aerodynamic and size specifications for a diverse variety of vehicles, such as trains and aircrafts.

The quest for affordable antenna solutions responding to all these needs is still at an early stage. The costs and complexity of phased array antennas at Ka-band do not fit most civil applications. Fully mechanically steerable antennas rely on mature technologies but their volume and maintenance costs make them unattractive. The development of antennas for Satcom terminals is therefore moving toward hybrid electromechanical solutions.

Continuous transverse stub (CTS) arrays [1]-[8] stand out among other antenna technologies for their stable active impedance against both frequency and scan angle. A seriesfed CTS antenna consists of a linear array of non-resonant long slots or extruding transverse stubs realized on the upper plate of a parallel-plate waveguide (PPW) launcher, which is fed by a primary line source. They typically support only the fundamental transverse electromagnetic (TEM) PPW mode. The antenna beam can be steered by changing the orientation of the line source with respect to the radiating elements. The line source can be controlled electronically, e.g. by means of small phased arrays [1] or switching among several feeds in a quasi-optical beamformer [1], [9], [10]. Alternatively, PPW feeding structures and radiating elements can be mutually rotated, as in variable inclined CTS (VICTS) arrays [1], [3]. Despite the extremely thin profile, series-fed CTS arrays are limited in bandwidth as they are traveling-wave antennas. Parallel-fed CTS architectures [5], [6], [11] were proposed for wideband performance. They are based on quasi-TEM corporate-feed networks [11], [12] exciting in phase an array of long slots.

However, all standard CTS architectures are inherently linearly-polarized. A common approach for manipulating the field radiated by CTS arrays consists in adding external polarization converters [13]-[15] above the radiating aperture. These devices increase the volume and may penalize the antenna performance. Moreover, they often need to be associated to servomotors for reconfiguring both the polarization and the scan angle.

A few series-fed CTS architectures achieving polarization agility have been recently presented in the literature [7], [8]. In these works, a shared aperture of cross-connected long slots is fed by two beamformers, launching quasi-plane TEM waves. A $90^{\circ}$ coupler is used for feeding the two beamformers and attaining dual-circular polarization in [7], while dual- 
linear polarization is demonstrated in [8]. Beside the increased size due to the additional beamformer, these solutions are extremely narrowband due to the traveling-wave operation.

To the authors' knowledge, this two-part paper presents for the first time a parallel-fed CTS antenna which achieves dual linear polarization using two excitation modes. The proposed approach radically differs from those previously described. Exciting a parallel-fed CTS array with two orthogonally polarized modes is a non-trivial task. As proved in this work, the simple strategy of replacing single-mode PPWs by overmoded ones does not ensure the correct antenna operation, due to modal coupling. Indeed, in an overmoded PPW (OPPW) supporting the first higher modes, i.e. the first transverse electric $\left(\mathrm{TE}_{1}\right)$ and transverse magnetic $\left(\mathrm{TM}_{1}\right)$ modes, the fundamental TEM mode can couple to the $\mathrm{TM}_{1}$ mode at any discontinuities.

The solution here proposed to attain dual-polarization capability relies on the use of corrugated parallel-plate waveguides (CPPWs) [16]-[19]. Each CPPW comprises two parallel plates, spaced at a distance between $\lambda / 2$ and $\lambda$, where $\lambda$ is the guided wavelength at the operating frequency, and two parallel rows of ridges, one on each plate. The propagation characteristics are investigated and engineered. Guidelines for minimizing the coupling between quasi-TEM and quasi-TM $\mathrm{TM}_{1}$ modes are presented. With this design, an ideal CPPW supports only a quasi-TEM and quasi-TE 1 mode, whose transverse electric fields are orthogonally polarized.

Based on this building block, a high-gain dual-polarized parallel-fed CTS array excited by a CPPW feed network is designed for Ka-band applications. We prove that, devising the modal dispersion in the CPPW structures, fractional bandwidths greater than $20 \%$ can be covered. That represents a tenfold enhancement with respect to current dual-polarized CTS architectures relying on cross-connected arrays. Dual-linear and dual-circular polarization can be obtained by properly feeding the antenna using one or both modes.

The present paper is organized as follows. Section II demonstrates that OPPWs are not suitable for designing bimodal corporate-feed networks for CTS arrays. In Section III, the proposed CPPW is described, its dispersion characteristics are discussed, with emphasis on Ka-band applications. The design of a Ka-band dual-polarization eight-slot CTS array is illustrated in Section IV as well as its matching performance. Radiation patterns and gain are shown in Section V. Conclusions are drawn in Section VI. The characterization of the design described in this paper, in combination with pillbox beamformers, is presented in Part II.

\section{LIMITATIONS OF OVERMODED PPWS IN DUAL-MODE CTS DESIGNS}

The orientation and distribution of the electric fields in the transverse section of an ideal PPW are illustrated in Fig. 1. The distance between the parallel perfectly conductive plates is $h$. The structure is infinite along $y$-axis and the fields propagate along $z$-direction. The propagating constants $k_{z n}$ of the $\mathrm{TE}_{n}$ mode and of the $\mathrm{TM}_{n}$ one are equal and, for any $n \in \mathbb{N}^{+}$, given by [20]

$$
k_{z n}=\sqrt{k^{2}-\left(\frac{n \pi}{h}\right)^{2}},
$$

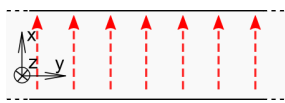

(a)

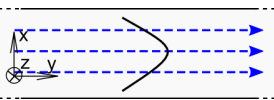

(b)

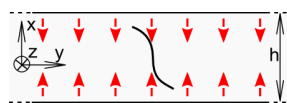

(c)
Fig. 1. Transverse electric field distributions of the first three modes in an ideal PPW. (a) TEM mode. (b) $\mathrm{TE}_{1}$ mode. (c) $\mathrm{TM}_{1}$ mode. The parallel plates are perfectly conducting planes, spaced apart at a distance $h$.

where $k=2 \pi \sqrt{\epsilon_{r}} / \lambda_{0}$ is the wavenumber in the dielectric medium of relative permittivity $\epsilon_{r}$ inside the PPW. It follows from (1) that if the distance $h$ is between $\lambda / 2$ and $\lambda$, where $\lambda=\lambda_{0} / \sqrt{\epsilon_{r}}$ is the wavelength at the frequency $f_{0}$, three modes propagate [20] at $f_{0}$ : the TEM, the $\mathrm{TM}_{1}$ and the $\mathrm{TE}_{1}$. The TEM mode and the $\mathrm{TM}_{1}$ one are polarized along the $x$-direction but have different distributions, shown in Fig. 1a and Fig. 1c, respectively. The amplitude of the first is constant, while that of the second varies as a cosine between the plates. The $\mathrm{TE}_{1}$ mode is oriented along the $y$-axis and its transverse electric field has a sinusoidal distribution along $x$-axis (see Fig. 1b).

A possible strategy for designing an OPPW network supporting orthogonally polarized modes is analyzed. It consists in exciting TEM and $\mathrm{TE}_{1}$ modes in a PPW with $\lambda_{L}^{O P P W} / 2<$ $h<\lambda_{H}^{O P P W}$, where $\lambda_{L}^{O P P W}$ and $\lambda_{H}^{O P P W}$ are the guided wavelengths at the lowest and highest frequency in the band, respectively. The transverse electric field of the $\mathrm{TE}_{1}$ mode is orthogonal to those of TEM and $\mathrm{TM}_{1}$ modes and its propagation can be thus analyzed independently of the other two. On the other hand, the TEM mode can significantly couple to the $\mathrm{TM}_{1}$ mode at any discontinuity, such as a Tjunction, since the transverse electric fields of these two modes are parallel. Such a modal coupling can hinder the operation of the power dividers in parallel-fed CTS architectures. To prove that, the behavior of the overmoded T-junction shown in Fig. 2 is investigated. The three OPPWs forming the junction are equal $(h=7 \mathrm{~mm})$. The structure is assumed infinite along $y$-axis. Accordingly, either perfect magnetic conductor (PMC) or perfect electric conductor (PEC) boundaries are enforced on the faces of the junction parallel to $x z$-plane for analyzing the component when it is fed by TEM and $\mathrm{TE}_{1}$ modes (see Fig. 2). Figure 3 a presents the simulated magnitude of the electric field, at $30 \mathrm{GHz}$, in the cross section of the T-junction, when Port 1 is excited by a TEM mode. The field distribution of a TEM mode can be clearly distinguished all along the input PPW, till the junction. However, a different field pattern is obtained at the output ports: that is a superposition of TEM and $\mathrm{TM}_{1}$ modes. The modal coupling can significantly degrade the input matching, phase response and frequency behavior of the T-junction as compared to the single TEM mode operation.

In the second case (see Fig. 3b), the input port is fed by a pure $\mathrm{TE}_{1}$ mode and the field pattern at the output ports is almost unperturbed. This field analysis demonstrates that standard OPPWs are not suitable for ensuring the dual-mode operation of dual-polarized CTS array, due to the partial conversion of the TEM mode into a $\mathrm{TM}_{1}$ mode in the $\mathrm{T}$ junctions of the feed network. 


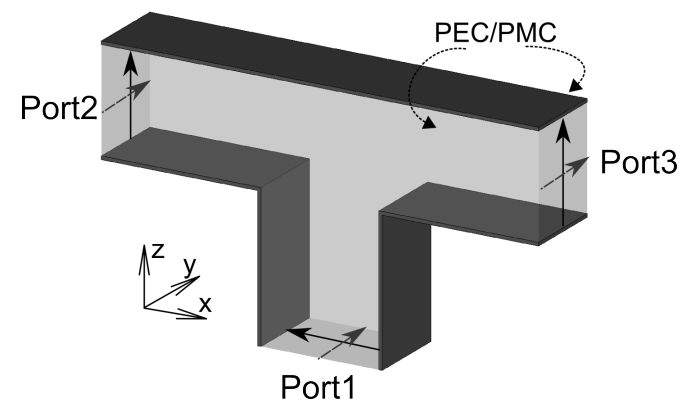

Fig. 2. View of the simulated T-junction among OPPWs. All OPPWs have the same height $h=0.7 \lambda_{0}$. The structure is infinite along $y$-axis and has been modeled using PMC or PEC boundaries on the faces parallel to the $x z$-plane for exciting $\mathrm{TEM} / \mathrm{TM}_{n}$ modes and $\mathrm{TE}_{n}$ modes, respectively.

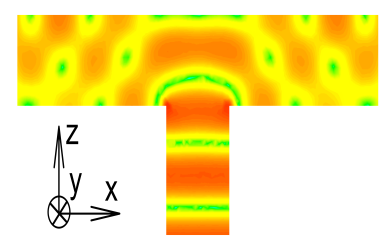

(a)

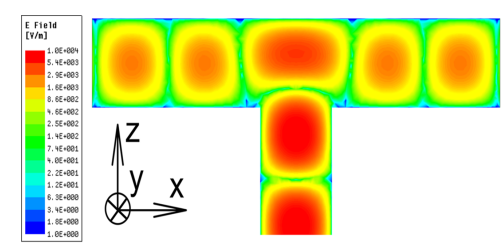

(b)
Fig. 3. Simulated amplitude of the electric field at $30 \mathrm{GHz}$ in the cross section of a T-junction among three ideal OPPWs of height $7 \mathrm{~mm}$, when the input port is fed by: (a) a TEM; (b) a $\mathrm{TE}_{1}$ mode. The results are obtained from full-wave simulations performed using Ansys Electronics Desktop 2018 and the boundary conditions shown in Fig. 2.

\section{BIMODAL CORRUGATED PARALLEL-PLATE WAVEGUIDE}

A novel bimodal CPPW is here proposed as a building block to realize CTS antennas achieving dual-linear or circular polarization. The CPPW is designed to support the two first orthogonally polarized modes, i.e. a quasi-TEM and a quasi$\mathrm{TE}_{1}$ mode, while preventing the propagation of the quasi$\mathrm{TM}_{1}$ mode and higher-order modes. The suppression of the quasi- $\mathrm{TM}_{1}$ mode is achieved by introducing corrugations on the outer plates of the OPPW analyzed in Section II. These corrugated plates are designed to behave as continuous walls for x-polarized fields. They delimit a waveguide region where the only supported $\mathrm{x}$-polarized transverse field is that of the quasi-TEM mode.

A parametric analysis of the dispersion properties of the CPPW is presented to prove that the propagation characteristics of the two supported modes can be essentially decoupled and that all TM modes are evanescent, even in the presence of discontinuities, e.g. T-junctions.

\section{A. Analysis of a $C P P W$}

Figure 4 shows a three-dimensional (3-D) view, transverse and longitudinal sections of a CPPW. The wave propagates along $z$-direction. The CPPW is assumed infinite along $y$ direction. The distance between the parallel plates is $h_{T E}$. The corrugation is realized introducing a periodic arrangement of ridges on each plate. The period is $p_{c}$. The corrugations on the two plates are identical and their distance along the $x$-axis is $h_{T E M}$. The section of the ridges is rectangular and the edges

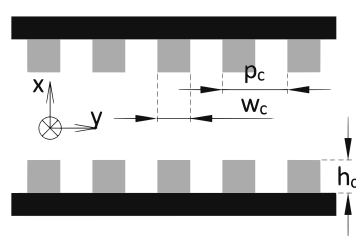

(a)

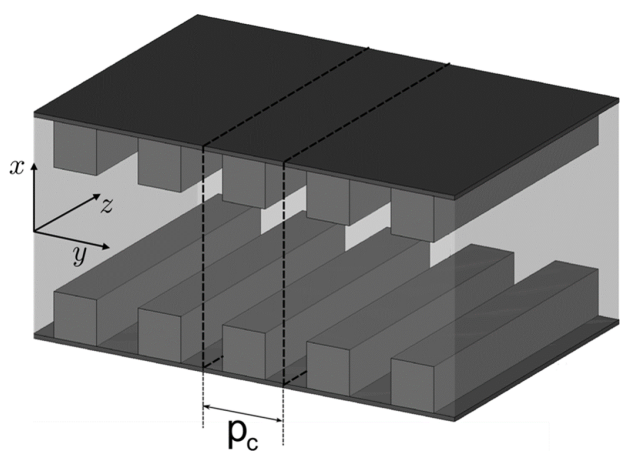

(c)

Fig. 4. (a) Transverse section, (b) longitudinal section and (c) 3-D view of the proposed corrugated parallel-plate waveguide.

have lengths $w_{c}$ and $h_{c}$. Such a CPPW supports only two orthogonally polarized modes in the frequency range between $f_{L}$ and $f_{H}$ provided that $p_{c}$ is electrically small, e.g. less than $\lambda_{H} / 5$ and that, at the same time, the following conditions are met

$$
\begin{gathered}
h_{T E M}<\lambda_{H} / 2 \\
\lambda_{L} / 2<h_{T E}<\lambda_{H}
\end{gathered}
$$

where $\lambda_{L}$ and $\lambda_{H}$ are the wavelength in the medium filling the CPPW at $f_{L}$ and $f_{H}$, respectively. In this situation, the parallel corrugations act for $x$-polarized fields, i.e. TEM- and TMlike modes, as effective conducting walls bounding the PPW region $-h_{T E M} / 2<x<h_{T E M} / 2$. The quasi-TEM mode propagates in this region but, from (2), the quasi- $\mathrm{TM}_{1}$ mode is evanescent, thus avoiding undesired TEM-TM $\mathrm{TM}_{1}$ coupling. From (3), a quasi- $\mathrm{TE}_{1}$ mode, having a $y$-polarized transverse electric field, is also supported. The quasi-TE 1 field spreads in the entire CPPW whereas the quasi-TEM field does not propagate in the region between corrugations and continuous plates, i.e. $h_{T E M} / 2<|x|<h_{T E} / 2$.

These qualitative observations can be rigorously demonstrated performing a dispersive analysis of a CPPW. A CPPW line fulfilling (2)-(3) between $f_{L}=27 \mathrm{GHz}$ and $f_{H}=32$ $\mathrm{GHz}$ is considered. The parameters of the CPPW are given in the caption of Fig. 5. The dispersion diagrams along the propagation axis of the first two modes of the CPPW, i.e. the quasi-TEM mode and the quasi-TE $\mathrm{TE}_{1}$ one, are compared to the analogous curves for TEM and $\mathrm{TE}_{1}$ modes in a PPW of height $h_{T E}$. The results for the CPPW were obtained using the eigenmode solver of Ansys Electronics Desktop 2018, while the curves relative to the PPW were computed using (1). The dispersion curve of the quasi-TEM mode in a CPPW matches that of a pure TEM mode in a PPW of height $h_{T E}$. Conversely, the quasi-TE $\mathrm{TE}_{1}$ mode in the CPPW and the $\mathrm{TE}_{1}$ 


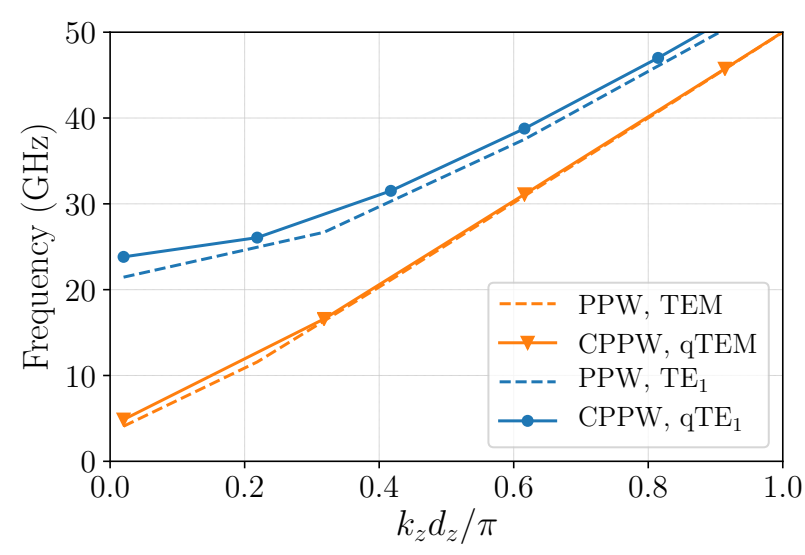

Fig. 5. Dispersion diagrams along the propagation axis $z$ of the CPPW of Fig. 4 and of the corresponding PPW with height $h_{T E}$. The geometrical parameters are: $h_{T E}=0.7 \lambda_{0}, h_{T E M}=0.4 \lambda_{0}, w=0.05 \lambda_{0}$ and $p_{c}=$ $0.15 \lambda_{0}$. The length of the CPPW along $z$-axis used in the full-wave simulation is $d_{z}=2 \lambda_{0}$. The results for the PPW are found using (1).

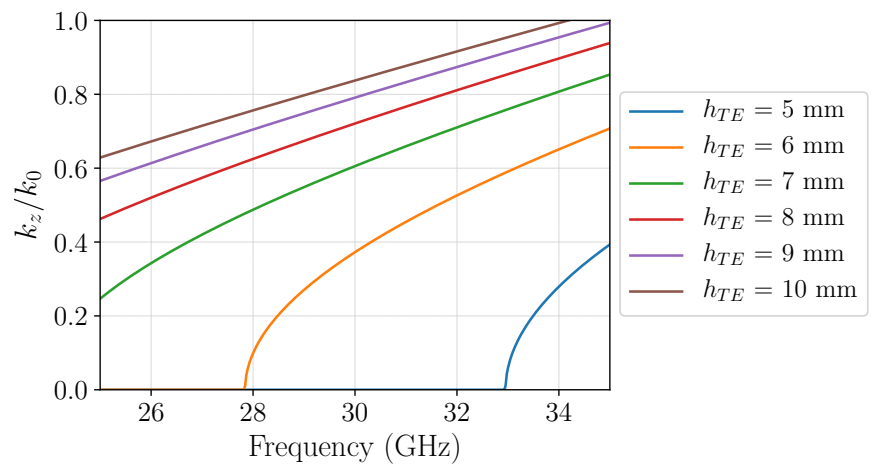

(a)

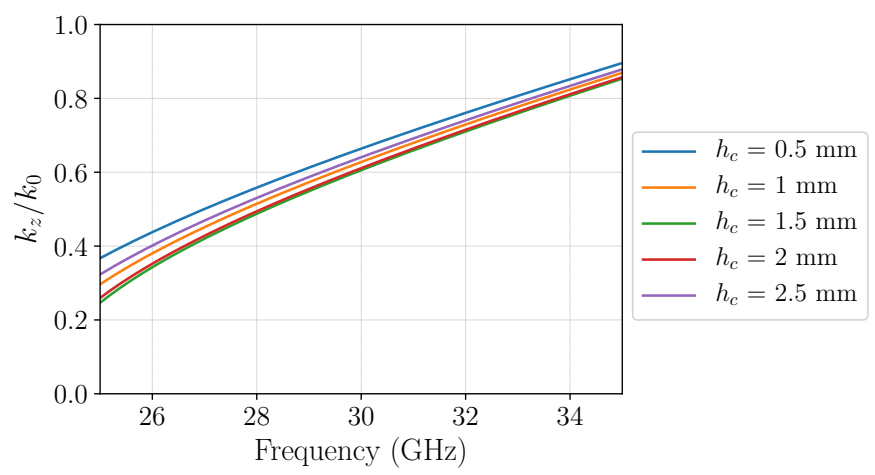

(b)

Fig. 6. Dispersion diagram of the quasi-TE $\mathrm{T}_{1}$ mode in a CPPW for normal incidence as a function of (a) $h_{T E}$, with $h_{c}=0.15 \lambda_{0}$; (b) $h_{c}$, with $h_{T E}=$ $0.7 \lambda_{0}$. The other parameters of the CPPW are $p_{c}=0.15 \lambda_{0}$ and $w_{c}=$ $0.05 \lambda_{0}$.

in the corresponding PPW have slightly different dispersive characteristics. Hence, as long as (2)-(3) hold, the corrugations of the proposed bimodal CPPW affect the propagation of the quasi-TE 1 mode while leaving unaltered the characteristics of the quasi-TEM mode as compared to an OPPW of height $h_{T E}$. This is a valuable property as it allows the designer to engineer the dispersion of the quasi- $\mathrm{TE}_{1}$ mode independently of the quasi-TEM mode. For instance, the slope of the dispersion curves of the two modes can be equalized for achieving

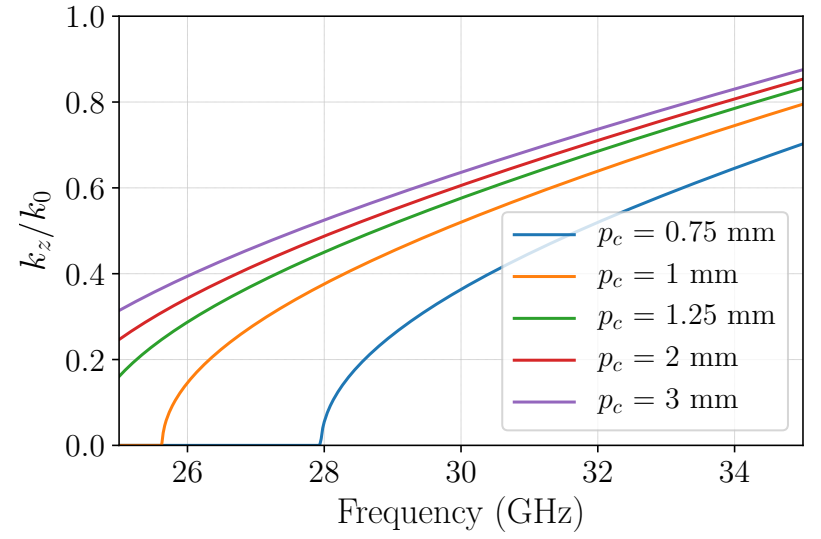

(a)

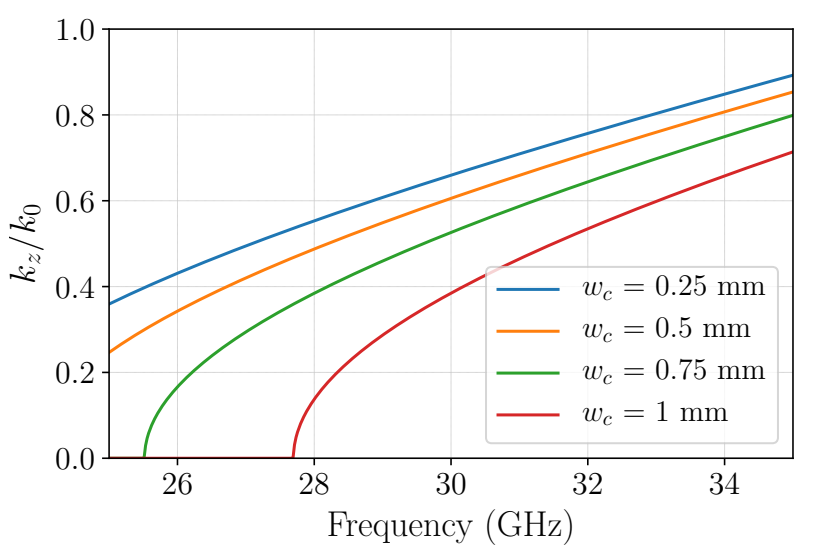

(b)

Fig. 7. Dispersion diagram of the quasi-TE $\mathrm{T}_{1}$ mode in a CPPW for normal incidence as a function of (a) $p_{c}$, for $w_{c}=0.5 \mathrm{~mm}$ and (b) $w_{c}$, for $p_{c}=1.5$ $\mathrm{mm}$. The other CPPW parameters are: $h_{T E M}=4 \mathrm{~mm}, h_{T E M}=7 \mathrm{~mm}$.

broadband operation.

The impact of parameters of the corrugations on the dispersion curve of the quasi-TE $\mathrm{TE}_{1}$ mode in the CPPW is analyzed in Fig. 6 and in Fig. 7. All dispersion diagrams refer to the propagation axis and were extracted from the simulated scattering parameters [20] of a unit cell of a hollow CPPW line. The dispersion curves of the quasi- $\mathrm{TE}_{1}$ mode are plotted in Fig. 6a for various values of the distance $h_{T E}$ between the outer plates. As in standard PPWs, the cut-off frequency rapidly decreases when $h_{T E}$ increases. Instead, for a given $h_{T E}$, the height $h_{c}$ of the corrugation does not significantly modify the dispersion characteristics (see Fig. 6b), as long as $h_{T E M}=h_{T E}-2 h_{c}<\lambda_{0} / 2$. This further proves that, within the latter range, the dispersion curve of the quasi- $\mathrm{TE}_{1}$ mode (only slightly dependent on $h_{T E M}$ ) and that of the quasiTEM mode, exclusively controlled by $h_{T E M}$, can be tailored independently.

Figure $7 \mathrm{a}$ shows the dispersion curves of the quasi- $\mathrm{TE}_{1}$ mode in a CPPW for several values of the period $p_{c}$ of the corrugation. The other parameters are listed in the caption. The cut-off frequency of the quasi- $\mathrm{TE}_{1}$ mode increases as $p_{c}$ decreases. One can intuitively explain this trend considering that, as $p_{c}$ decreases, the corrugated walls of the CPPW tend to become uniform surfaces bounding a single-mode PPW. 


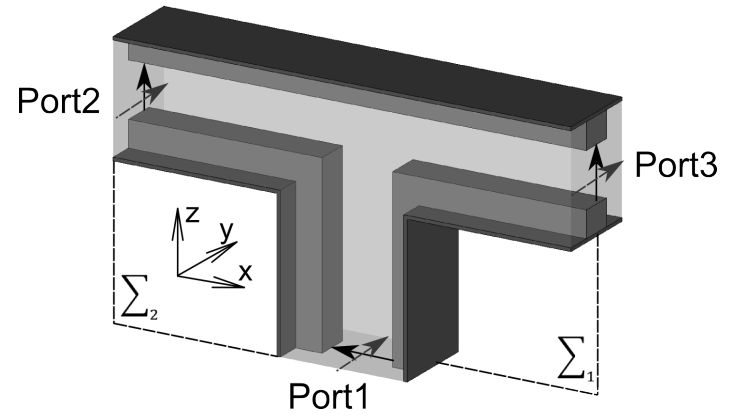

Fig. 8. Geometry of a T-junction among CPPWs. The TEM/TM 1 and $\mathrm{TE}_{1}$ modes are excited using the boundary conditions of Fig. 2. Input and output CPPWs have the same geometrical parameters: $h_{T E}=0.7 \lambda_{0}, h_{T E M}=$ $0.4 \lambda_{0}, p_{c}=0.15 \lambda_{0}$ and $w_{c}=0.05 \lambda_{0}$.

Similar arguments help to interpret the results in Fig. 7b: for a fixed period, the cut-off frequency increases with the width $w_{c}$ of the ridges.

\section{B. Suppression of TM modes in the presence of discontinuities}

The results presented in Section III-A proved that a CPPW can be designed to support two orthogonally polarized modes in a given band. For a successful use of CPPWs in dual-mode parallel-fed CTS antennas, no significant mode conversion should occur in the power dividers. The 3-D view of a basic T-junction among CPPWs is shown in Fig. 8. For simplicity, no matching structures are added. Input and output arms are identical as in standard CTS designs [11].

It has been shown in Section II that when a pure TEM mode feeds one port of a T-junction among OPPWs, it excites a non-decaying $\mathrm{TM}_{1}$ mode. Such a modal coupling does not occur in a T-junction among bimodal CPPWs. Figure 9 shows the amplitude of the electric field in the junction at $30 \mathrm{GHz}$ when the input CPPW (Port 1 in Fig. 8) is fed by a quasiTEM mode. In particular, Fig. 9a shows the field distribution in the vertical cut plane $\Sigma_{1}$ (see Fig. 8) parallel to the $x z$ plane and intersecting the centerline of the corrugated walls. Figure $9 \mathrm{~b}$ shows the same quantity in a plane that does not pass through the ridges (section $\Sigma_{2}$ in Fig. 8). The simulated device is infinite along the $y$-direction. Even if a TM mode is excited in the T-junction, it decays at the outputs of the junction since, by virtue of (2), it is not supported in the waveguide region bounded by the corrugated surfaces, which act as uniform plates for $\mathrm{x}$-polarized fields. A quasi-TEM mode distribution is therefore observed at the output ports (see Fig. 9a). Moreover, it is clear from Fig. 9b that the quasiTEM mode is well confined in the inner waveguide region bounded by the corrugated surfaces. The amplitude of the electric field in the same cut planes when the input port is fed by a quasi-TE ${ }_{1}$ mode is plotted in Fig. 10. As in the Tjunction among OPPWs analyzed in Section II, no other modes are excited. Furthermore, Fig. 10b proves that the quasi-TE 1 mode spreads in the entire CPPW, i.e. the electric field has a significant amplitude also in the regions among the ridges of the corrugations.

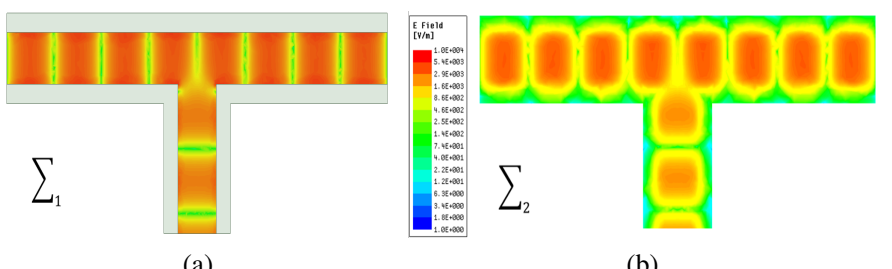

(a)

(b)

Fig. 9. Simulated amplitude of the electric field at $30 \mathrm{GHz}$ when the T-junction of Fig. 8 is excited by a quasi-TEM mode at Port 1. Field plot at section: (a) $\Sigma_{1}$ and (b) $\Sigma_{2}$.

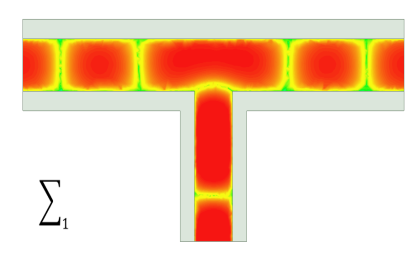

(a)

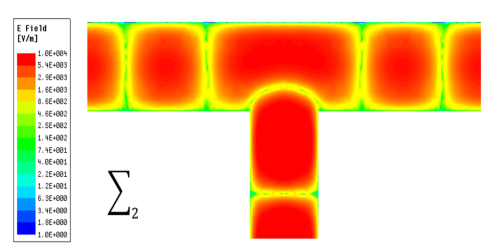

(b)
Fig. 10. Simulated amplitude of the electric field at $30 \mathrm{GHz}$ when the Tjunction of Fig. 8 is excited by a quasi-TE 1 mode at Port 1 . Field plot at section: (a) $\Sigma_{1}$ and (b) $\Sigma_{2}$.

The simulated transmission coefficients of the T-junction (from Port 1 to Port 2) are plotted in Fig. 11 and Fig. 12 to provide a more quantitative view of the modal coupling. The same port impedance has been used in simulations for input and output arms. The results were post-processed removing the impact of the impedance mismatch at the ports [20]. Port 1 is excited either by a quasi-TEM mode or by a quasi-TE $\mathrm{T}_{1}$ mode. For a quasi-TEM excitation, two coefficients are monitored, expressing the power carried on Port 2 by the quasi-TEM mode and quasi- $\mathrm{TM}_{1}$ mode, respectively. The latter coefficient is a metric of the TEM-to-TM mode coupling. Figure 11 presents the simulated coefficients at $30 \mathrm{GHz}$ as functions of the height $h_{T E}$ of the CPPWs. The height $h_{c}=0.15 \lambda_{0}$ of the ridges is fixed and thus the distance $h_{T E M}=h_{T E}-2 h_{c}$ varies with $h_{T E}$. The quasi-TE 1 mode is transmitted to Port 2 with negligible loss for $h_{T E}$ slightly higher than the cut-off value, corresponding to half a wavelength. The quasi-TEM mode propagates without coupling to the quasi-TM $\mathrm{TM}_{1}$ up to the threshold value $h_{T E}=0.77 \lambda_{0}$. For greater distances of the plates, most of the power on the output port is carried by the quasi-TM $\mathrm{TM}_{1}$ mode and the quasi-TEM attenuates. The onset of the quasi-TM $\mathrm{TM}_{1}$ mode is related to the value of $h_{T E M}$, which exceeds half a wavelength when $h_{T E}>0.7 \lambda_{0}$.

The impact of $h_{c}$ is then studied in Fig. 12, fixing $h_{T E}=$ $0.7 \lambda_{0}$. The transmission coefficient of the quasi-TE $\mathrm{TE}_{1}$ is almost constant (about $-3 \mathrm{~dB}$ ) for any $h_{c}$. Conversely, the quasi-TEM mode does not couple to the quasi-TM $\mathrm{TM}_{1}$ only if $h_{c}>0.12 \lambda_{0}$. For lower values of $h_{c}$, the inner region bounded by the corrugations becomes large enough to support a quasi-TM mode, beside the quasi-TEM one.

\section{DuAl-POL CTS ARRAY DESIGN}

This section discusses the design and matching performance of a dual-pol CTS array of eight elements. The radiating 


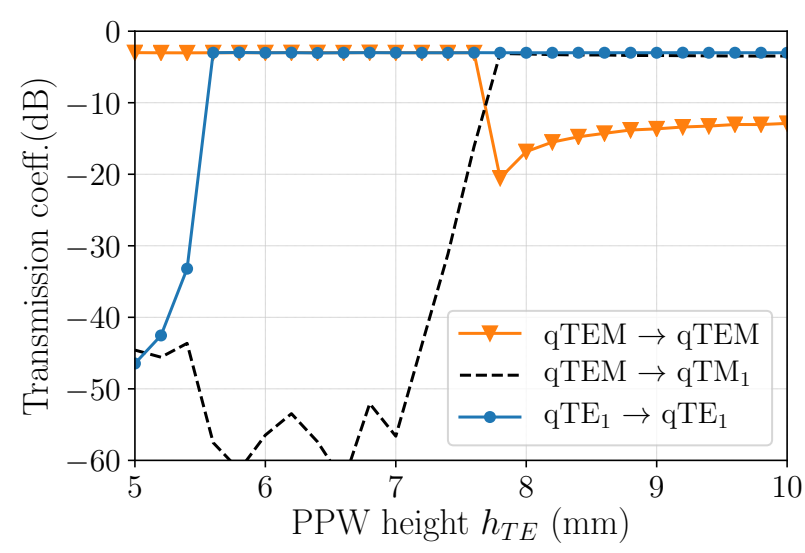

Fig. 11. Simulated transmission coefficients from the Port 1 to the each of the output ports of the CPPW T-junction shown in Fig. 8, as functions of $h_{T E}$, at $30 \mathrm{GHz}$. In the legend, the mode launched at Port 1 and that considered at the output port are on the left and right hand side of the arrow.

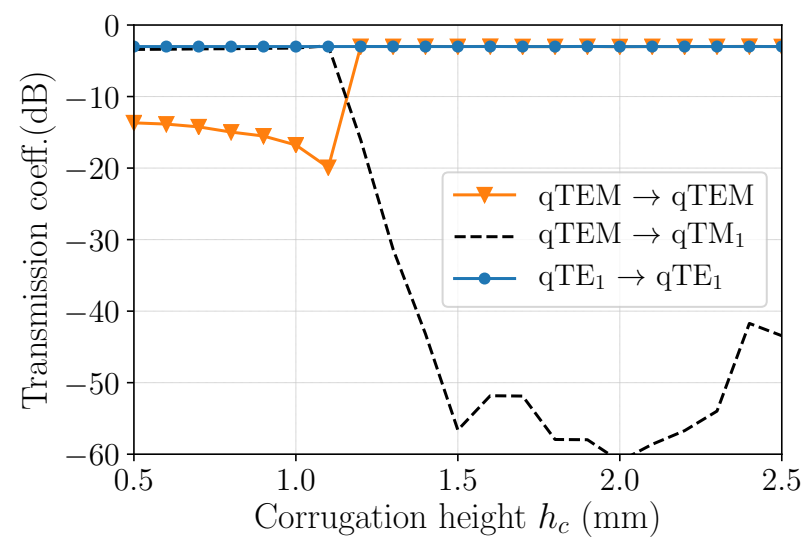

Fig. 12. Simulated transmission coefficients from Port 1 to each of the output ports of the CPPW T-junction shown in Fig. 8 as functions of $h_{c}$, at $30 \mathrm{GHz}$. In the legend, the mode launched at Port 1 and that considered at the output port are on the left and right hand side of the arrow.

elements are overmoded long slots excited by a one-to-eight corporate-feed network (CFN) supporting quasi-TEM and quasi- $\mathrm{TE}_{1}$ modes. The cross-section of the antenna is shown in Fig. 13. The inner regions of the CPPW components, bounded between the corrugations, are shown in white and can be seen as the equivalent waveguide for the quasi-TEM mode. The regions occupied by the ridges are instead shown in gray. The reference CPPW, used as input line and output lines of all power dividers, has the following parameters: $h_{T E M}=0.4 \lambda_{0}$ $=4 \mathrm{~mm}, h_{T E}=0.7 \lambda_{0}=7 \mathrm{~mm}, h_{c}=0.15 \lambda_{0}=1.5 \mathrm{~mm}$, $p_{c}=0.15 \lambda_{0}=1.5 \mathrm{~mm}$ and $w_{c}=0.05 \lambda_{0}=0.5 \mathrm{~mm}$. This design was chosen on the basis of the study presented in Section III to attain a pure bimodal regime between $f_{L}=$ $27 \mathrm{GHz}$ and $f_{H}=32 \mathrm{GHz}$. Moreover, as shown in Fig. 5, the selected parameters provide similar dispersive behaviors for the two modes within the band.

\section{A. Long slot array}

The geometry of the array element is illustrated in Fig. 14. A tapered transition from CPPW to OPPW is designed to feed each slot. Thanks to this choice, the radiating apertures of the long slots are the same when excited by both modes. Thus,

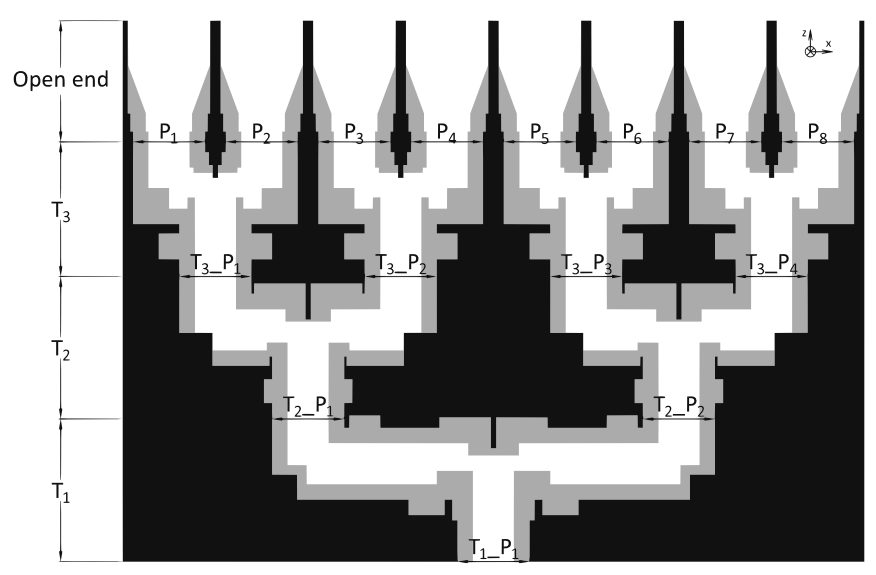

Fig. 13. Cross-section of the proposed Ka-band dual-polarized CTS antenna. The corporate-feed network is realized using dual-mode CPPWs. The regions occupied by corrugations are shown in gray.

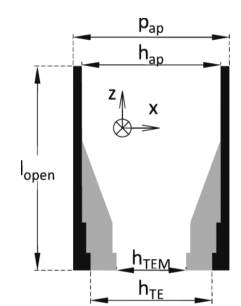

(a)

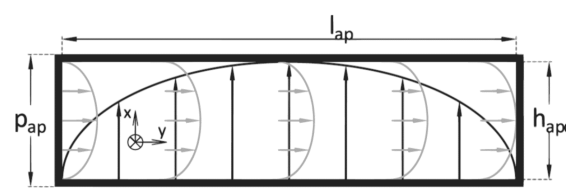

(b)
Fig. 14. Geometry of the radiating slots. (a) Cross section view and matching structure. (b) Top view and electric field profiles of quasi-TEM mode (in black) and quasi- $\mathrm{TE}_{1}$ mode (in gray).

the difference of directivity in the two polarization modes is minimized. If open-ended CPPWs were selected as radiating elements, the quasi-TEM mode would have been confined between the corrugated surfaces, i.e. in a transverse section smaller than that occupied by quasi- $\mathrm{TE}_{1}$ mode, yielding a lower directivity. The width of the slots was set to $h_{a p}=0.8 \lambda_{0}$ $=8 \mathrm{~mm}$ to maximize the radiating apertures without altering the bimodal regime of the CPPW feeds. The array spacing plays a key role in the frequency behavior of the active impedances [21] of the array elements. As a first step, the numerical tool presented in [4] was employed for analyzing the the active impedance of an infinite array of infinitely long slots as a function of $p_{a p}$. Figure 15a shows the active reflection coefficient [21] of the broadside array fed by a TEM mode, for several values of the period. For $p_{a p}=10 \mathrm{~mm}\left(\lambda_{0}\right.$ at 30 $\mathrm{GHz}$ ), the onset of a grating lobe at boresight determines a peak in the reflection coefficient. This peak moves out of the band of interest for smaller array spacing, e.g. $p_{a p}=0.9 \lambda_{0}$ $=9 \mathrm{~mm}$. Figure $15 \mathrm{~b}$ shows the active reflection of the array under $\mathrm{TE}_{1}$ mode excitation, for the same values of $p_{a p}$. The value $p_{a p}=0.9 \lambda_{0}$ was selected to get low and slowlyvarying reflection coefficients in the $27-35 \mathrm{GHz}$ band for both excitation modes. The impedances of the radiating elements were then matched to the output CPPW lines of the power dividers (ports P1 to P8 in Fig. 13) using the CPPW-to-OPPW transition shown in Fig. 14a. The active reflection coefficients at ports P1 - P4, for both excitation modes, are plotted in Fig.

\section{ACCEPTED MANUSCRIPT}




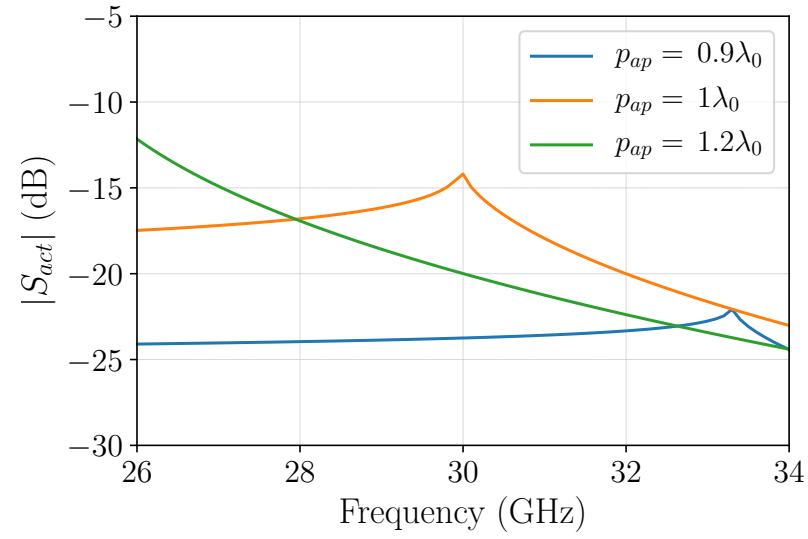

(a)

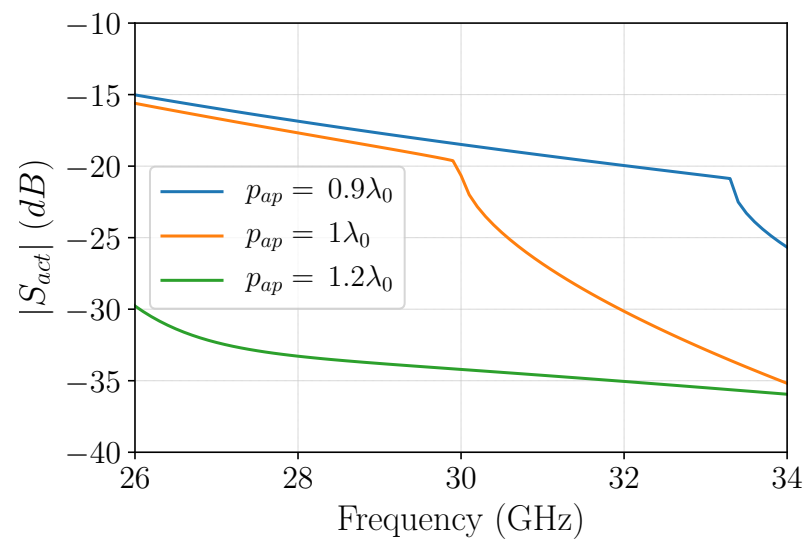

(b)

Fig. 15. Simulated active reflection coefficient of an infinite broadside array of slots as a function of frequency for several values of the array period $p_{a p}$. The slots are infinitely extended along y-axis and their width is $h_{a p}=0.8 \lambda_{0}$ $=8 \mathrm{~mm}$. Results for: (a) TEM and (b) $\mathrm{TE}_{1}$ input PPW modes.

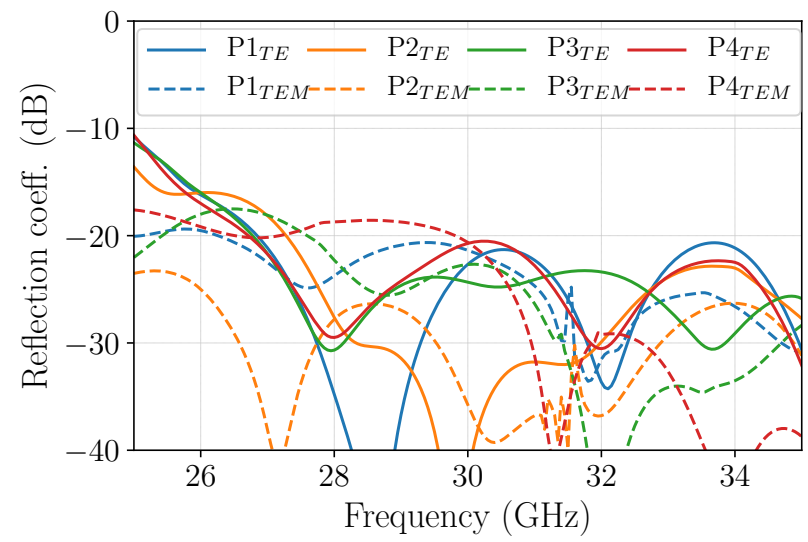

Fig. 16. Active reflection coefficients of the radiating elements after adding the matching structures of Fig. 14a, when excited by a quasi-TEM mode and a quasi-TE 1 mode. The reference sections are placed at the output ports of power dividers in the last division stage (ports P1 - P8 in Fig. 13). By virtue of symmetry, only the results for ports P1 - P4 are shown.

16. These results were obtained from full-wave simulations of the array of eight slots fed by the transitions, assuming the structure infinite along $y$-axis. The return losses at ports P5 - P8 are equal to those at ports P1 - P4, by virtue of symmetry with respect to $y z$-plane (see Fig. 13). All reflection coefficients are $<-15 \mathrm{~dB}$ between $26 \mathrm{GHz}$ and $35 \mathrm{GHz}$. Their variations across the ports are small. Finally, the length $l_{a p}$ of the slots is fixed to $30 \lambda_{0}=300 \mathrm{~mm}$ for achieving a directivity higher than $33 \mathrm{dBi}$ in the design band $(27-31 \mathrm{GHz})$.

\section{B. Corporate-feed network}

The feed network comprises three stages of balanced power dividers. The power dividers in the same division stage are equal (see Fig. 13). A reflection coefficient lower than $-15 \mathrm{~dB}$ between 27 and $32 \mathrm{GHz}$ was targeted at the input of each power divider. The output ports of each divider are terminated by the same impedances and the structures are infinite along $y$-axis. As shown later, the variation of the active impedance with the radiating element (see Fig. 16) and the finiteness of the CPPWs have no relevant impact on the input matching.

The conceptual view of a CPPW as the superposition of two effective PPWs, one for each mode, inspired the design approach. As a first step, the basic designs of the splitters were defined for a quasi-TEM excitation, assuming effective PPWs of height equal to the distance between the corrugated surfaces ( $h_{T E M}$ in Fig. 4). This preliminary design was finalized using transmission line models [22] and following the guidelines in [11], [23]. Then, the distances between the outer walls of the CPPWs were designed for each component with the aid of full-wave simulations and matching elements were introduced to enhance the performance under quasi- $\mathrm{TE}_{1}$-mode excitation. The assumption of infinite length along $y$-axis significantly reduces the computation time. The main parameters of the feed network are listed in Table 1.

The last division stage comprises four power dividers. Their design is shown in Fig. 17a. The edge-to-edge distance of the output ports is $p_{a p}-h_{T E}=2 \mathrm{~mm}$. A septum-like structure is introduced between the output ports for matching the power divider when fed by a quasi- $\mathrm{TE}_{1}$ mode. The simulated active reflection coefficients at ports T3_P $\mathrm{P}_{1}-\mathrm{T} 3 \mathrm{P}_{4}$ (see Fig. 13) are $<-15 \mathrm{~dB}$ between $27 \mathrm{GHz}$ and $32 \mathrm{GHz}$, for both input modes.

The design of the power dividers in the second stage is shown in Fig. 17b. These dividers are matched using quarter wavelength transformers and steps in the bends. Narrow insets of widths $<\lambda_{0} / 10$ (post $_{T E \text { Ein }}$ and post $_{T E o u t}$ ) are introduced for enhancing the matching under quasi-TE $\mathrm{T}_{1}$ mode excitation. The simulated active reflection coefficients at the input ports of these power dividers are both less than $-20 \mathrm{~dB}$.

The input power divider is shown in Fig. 17c. As opposed to the other designs, there are no matching stubs along the input line. Instead, matching sections are added in the arms of the junction (see $s t u b_{T E M i n}$ and $s t u b_{T E i n}$ ).

Figure 18 shows the simulated reflection coefficients of the overall network. The results obtained enforcing infinite periodic boundary conditions along $y$-axis (see Fig. 13) are compared to simulations on the finite structure. The length of the designed CTS array along $y$-axis is $300 \mathrm{~mm}$, i.e. $200 p_{c}$. In this case, the network comprises overmoded rectangular waveguides, since the infinite CPPWs are truncated along $y$ direction and closed on metallic walls (on the $x z$-planes). The reflection coefficients found for finite and infinite structures are in good agreement, due to the large electrical length (30

\section{ACCEPTED MANUSCRIPT}




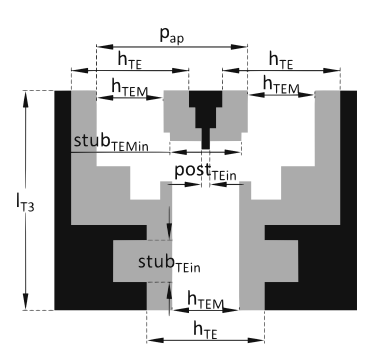

(a)

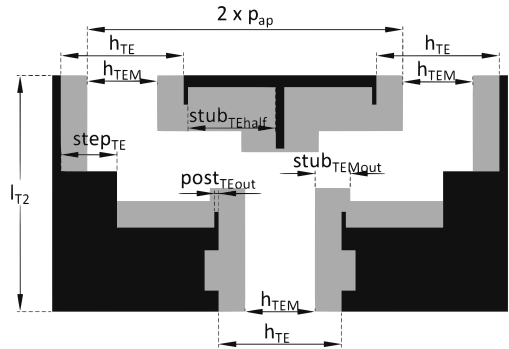

(b)

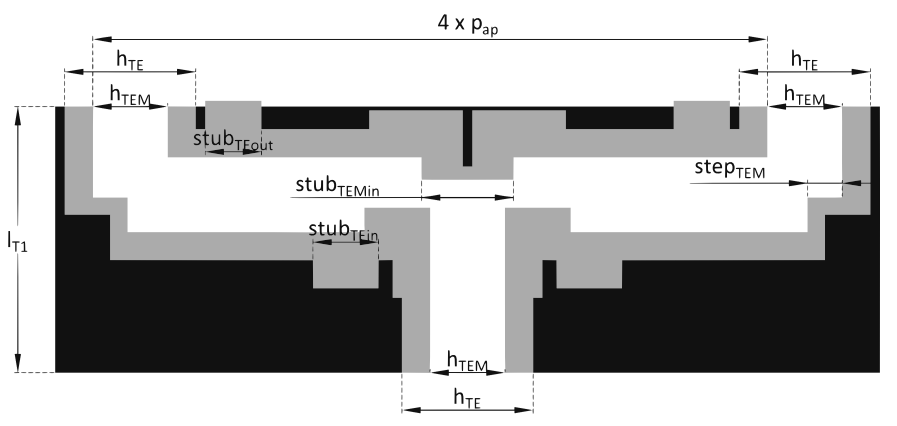

(c)

Fig. 17. Design and main geometrical parameters of the power dividers used in the three division stages of the feed network (see Fig. 13). (a) Last division stage (T3) with output ports spaced center to center by $p_{a p}$; (b) second division stage (T2); (c) first division stage (T1).

Table 1. MAIN PARAMETERS OF THE POWER DIVIDERS

\begin{tabular}{|c|c|c|c|}
\hline & T1 & $\mathrm{T} 2$ & T3 \\
\hline$l_{T}$ & $14.2 \mathrm{~mm}$ & $13.5 \mathrm{~mm}$ & $13 \mathrm{~mm}$ \\
\hline step $_{T E}$ & $2.42 \mathrm{~mm}$ & $3.2 \mathrm{~mm}$ & - \\
\hline step $_{T E M}$ & $1.85 \mathrm{~mm}$ & $1.7 \mathrm{~mm}$ & $2 \mathrm{~mm}$ \\
\hline$s t u b_{T E \text { in }}$ & $3.5 \mathrm{~mm}$ & $2.3 \mathrm{~mm}$ & $2.5 \mathrm{~mm}$ \\
\hline$s t u b_{T E M i n}$ & $4.9 \mathrm{~mm}$ & $4.4 \mathrm{~mm}$ & $4.25 \mathrm{~mm}$ \\
\hline stub $_{\text {TEout }}$ & $3 \mathrm{~mm}$ & - & - \\
\hline stub $_{\text {TE Mout }}$ & $3.5 \mathrm{~mm}$ & $2 \mathrm{~mm}$ & $0.7 \mathrm{~mm}$ \\
\hline$s^{\prime} u b_{T E h a l f}$ & $5 \mathrm{~mm}$ & $5 \mathrm{~mm}$ & - \\
\hline post $_{T E \text { En }}$ & $0.5 \mathrm{~mm}$ & $0.5 \mathrm{~mm}$ & $0.5 \mathrm{~mm}$ \\
\hline post $_{\text {TEout }}$ & - & $0.25 \mathrm{~mm}$ & - \\
\hline
\end{tabular}

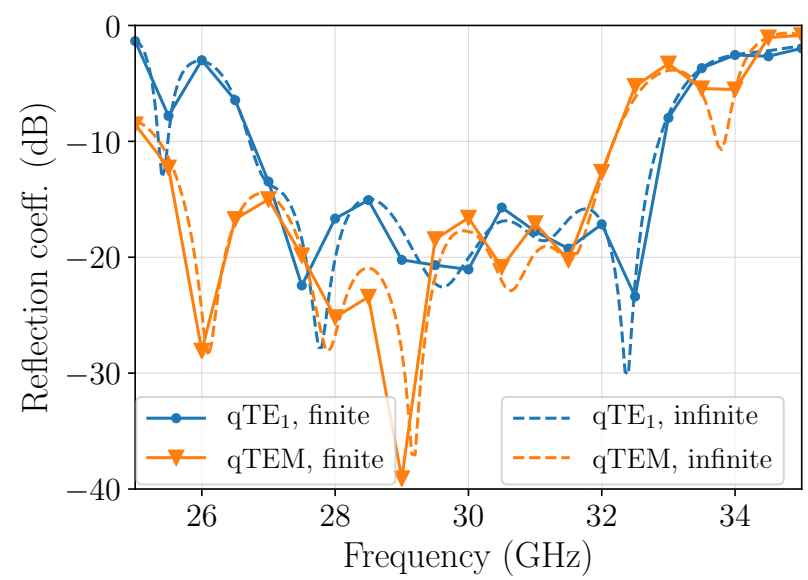

Fig. 18. Simulated reflection coefficients at the input of the CPPW feed network (T1_P1 in Fig. 13), for quasi-TEM and quasi-TE 1 -mode excitation. The results of the actual design are compared to those obtained assuming the structure infinite along $y$-axis.

$\lambda_{0}$ ) of the structure along $y$-axis. In the targeted band, the input reflection coefficient of the finite structure is $<-15 \mathrm{~dB}$ for the quasi- $\mathrm{TE}_{1}$ mode and $<-12 \mathrm{~dB}$ for the quasi-TEM one.

\section{RADIATION PERFORMANCE}

This section provides a numerical validation of the dualmode CTS antenna concept. It proves that the presented Ka-band design radiates horizontally $(\mathrm{H})$ or vertically $(\mathrm{V})$ polarized fields when excited by a quasi-TEM mode or a quasi$\mathrm{TE}_{1}$ mode, respectively. The normalized radiation patterns in the $y z$-plane (see Fig. 13) at $30 \mathrm{GHz}$ are plotted in Fig. 19. For both modes, pure linear polarization are achieved, with peak cross-polarization levels lower than $-50 \mathrm{~dB}$. Two methods were followed to compute gain and radiation patterns. The first is based on the numerical model developed in [4] for the analysis of an array of long slots. It resorts to a windowing approach [24]: the equivalent magnetic currents are calculated on a single unit cell, assuming that the array is infinite and periodic. The radiation patterns are then determined considering that a finite number of cells radiates. The second method consists in a full-wave simulation of the overall antenna, considering copper $\left(\sigma=5.8 \times 10^{7} \mathrm{~S} / \mathrm{m}\right)$ as conductive material. In simulations, the antenna is fed at the input port of the corporate-feed network, which accounts for finiteness of the array and of the feed network. The latter effects are not relevant in the proposed design, as demonstrated by the excellent agreement of the results. The first sidelobe levels (SLLs) are in the order of $-13 \mathrm{~dB}$ for both polarizations since the amplitude of the input source was not tapered in the $x z$-plane. A significant reduction of the SLLs can be achieved using, for instance, a parabolic pillbox beamformer [5], [6] to feed the antenna, as discussed in Part II of this paper. The realized gain of the dual-polarized CTS antenna is plotted as a function of frequency in Fig. 20. The simulated curves are compared, for both polarization, to the gain of the bare slot array which is computed using an inhouse script, without considering the corporate-feed network. Thus, the latter values represent upper bounds for the realized gain of the antenna. Between $27 \mathrm{GHz}$ and $32 \mathrm{GHz}$, the average differences between the gain of the bare array and the realized gain of the full CTS antenna are $0.2 \mathrm{dBi}$ under quasi-TE $\mathrm{T}_{1}$ mode excitation and $0.6 \mathrm{dBi}$ under quasi-TEM mode excitation. These small losses are due to the mismatch at the input of the antenna and to the ohmic loss of the feed network, which are not accounted by the script. The maximum difference between the realized gain values for $\mathrm{H}$ - and $\mathrm{V}$-polarizations is only $0.4 \mathrm{dBi}$, thanks to the shared aperture architecture and the good impedance matching attained for both modes. Between $27 \mathrm{GHz}$ and $32 \mathrm{GHz}$, i.e. in a band wider than that allocated for Satcom uplinks, the realized gain varies from $33.1 \mathrm{dBi}$ to $34.6 \mathrm{dBi}$ when the antenna is fed by a quasi- $\mathrm{TE}_{1}$ mode. A similar performance is observed for the other polarization. 


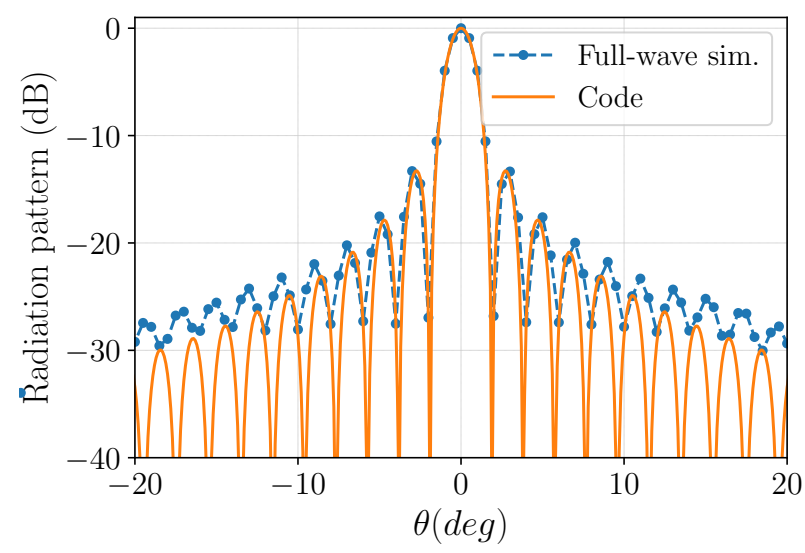

(a)

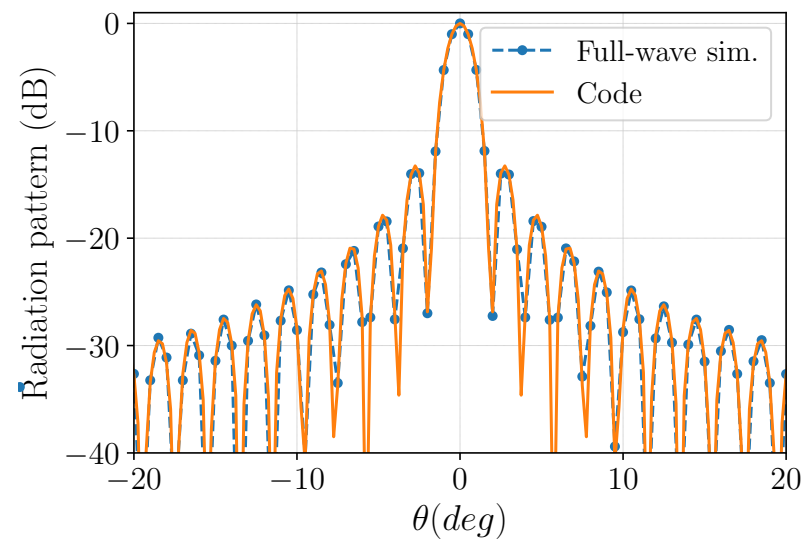

(b)

Fig. 19. Simulated radiation patterns (co-polar component, cut in the $x z$ plane) at $30 \mathrm{GHz}$ when the antenna is excited by (a) a quasi-TEM mode and (b) a quasi-TE mode. The results for the bare slot array, obtained using an in-house code, are compared to full-wave simulations of the entire antenna.

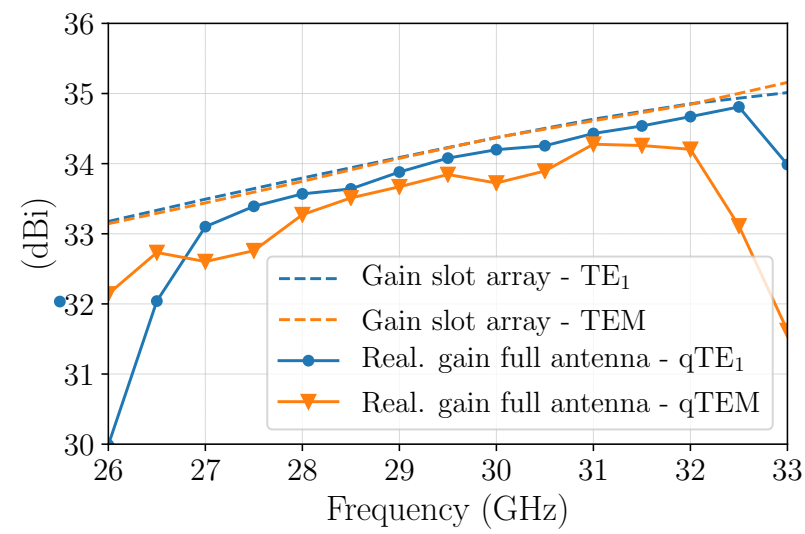

Fig. 20. Simulated realized gain against frequency of the full dual-polarized CTS antenna, when it is excited by a quasi-TEM and a quasi-TE ${ }_{1}$ mode, compared to the gain of the stand-alone slot array.

\section{CONCLUSION}

A novel parallel-fed dual-mode CTS architecture for dualpolarization applications has been presented. It has been proved that standard PPWs are unsuited for exciting the array with TEM and $\mathrm{TE}_{1}$ modes. Indeed, the TEM mode couples to the $\mathrm{TM}_{1}$ mode at any discontinuity in the feed network. A corrugated PPW has been proposed to ensure the propagation of only two orthogonal modes (quasi-TEM and quasi-TE ) $_{1}$ and to avoid the excitation of TM modes. The distance between the parallel plates of the CPPW is between $\lambda / 2$ and $\lambda$, where $\lambda$ is the guided wavelength. Two rows of ridges, one on each plate, define the two corrugated surfaces. The dispersion characteristics of the CPPW under normal incidence have been extensively analyzed and guidelines for achieving broadband bimodal operation have been drafted. In particular, conditions and strategies to independently devise the propagation of the two modes have been outlined. The design of a Ka-band CTS array of eight slots fed by a CPPW network has been described. As per simulations, when uniformly excited, the antenna achieves, for both polarizations, a relative $-3-\mathrm{dB}$ gain bandwidth exceeding $20 \%$, a gain of about $34 \mathrm{dBi}$ at $30 \mathrm{GHz}$ and a cross-polarization discrimination higher than $50 \mathrm{~dB}$.

The fabrication and characterization of the designed CTS antenna is presented in Part II. The dual-polarization capability and the scanning performance are experimentally validated by combining the antenna with pillbox beamformers.

\section{REFERENCES}

[1] W. W. Milroy, "The continuous transverse stub (CTS) array: basic theory, experiment, and application," in Proc. Antenna Appl. Symp., vol. 2, Sep. 1991, pp. 253-283.

[2] — "Continuous transverse stub element devices and methods of making same," U.S. Patent 5266 961, 11 30, 1993.

[3] W. W. Milroy, S. B. Coppedge, and A. C. Lemons, "Variable inclination continuous transverse stub array,” U.S. Patent 6919854 B2, 7 19, 2005.

[4] F. Foglia Manzillo, M. Ettorre, M. Casaletti, N. Capet, and R. Sauleau, "Active impedance of infinite parallel-fed continuous transverse stub arrays," IEEE Trans. Antennas Propag., vol. 63, no. 7, pp. 3291-3297, Jul. 2015.

[5] M. Ettorre, F. Foglia Manzillo, M. Casaletti, R. Sauleau, L. Le Coq, and N. Capet, "Continuous transverse stub array for Ka-band applications," IEEE Trans. Antennas Propag., vol. 63, no. 11, pp. 4792-4800, Nov. 2015.

[6] F. Foglia Manzillo et al., "A wide-angle scanning switched-beam antenna system in LTCC technology with high beam crossing levels for V-band communications," IEEE Trans. Antennas Propag., vol. 67, no. 1, pp. $541-553$, Jan. 2019.

[7] Y. J. Cheng, J. Wang, and X. L. Liu, "94 GHz substrate integrated waveguide dual-circular-polarization shared-aperture parallel-plate longslot array antenna with low sidelobe level," IEEE Trans. Antennas Propag., vol. 65, no. 11, pp. 5855-5861, Nov. 2017.

[8] T. Lou, X.-X. Yang, L. Li, and E. Abubaker, "A flat dual-polarized continuous transverse stub antenna array based on substrate integrated waveguide," in 12th Eur. Conf. Antennas Propag. (EuCAP), Apr. 2018, pp. $1-3$.

[9] M. Ettorre, R. Sauleau, and L. Le Coq, "Multi-beam multi-layer leakywave SIW pillbox antenna for millimeter-wave applications," IEEE Trans. Antennas Propag., vol. 59, no. 4, pp. 1093-1100, Apr. 2011.

[10] T. Teshirogi, Y. Kawahara, A. Yamamoto, Y. Sekine, N. Baba, and M. Kobayashi, "Dielectric slab based leaky-wave antennas for millimeter-wave applications," in Proc. IEEE Antennas Propag. Soc. Int. Symp., vol. 1, Jul. 2001, pp. 346-349.

[11] W. W. Milroy, "Compact, ultra-wideband, antenna feed architecture comprising a multistage, multilevel network of constant reflectioncoefficient components," U.S. Patent 6075 494, Jun. 13, 2000.

[12] W. W. Milroy, S. B. Coppedge, A. Ekmekji, S. Hashemi-Yeganeh, and S. G. Buczek, "True-time-delay feed network for CTS array," Patent US $7432871,107,2008$.

[13] A. Ericsson and D. Sjöberg, "Design and analysis of a multilayer meander line circular polarization selective structure," IEEE Trans. Antennas Propag., vol. 65, no. 8, pp. 4089-4101, Aug. 2017.

[14] F. Foglia Manzillo, M. Ettorre, R. Sauleau, and A. Grbic, "Systematic design of a class of wideband circular polarizers using dispersion engineering," in 11th Eur. Conf. Antennas Propag. (EuCAP), Mar. 2017, pp. $1279-1281$.

\section{ACCEPTED MANUSCRIPT}


[15] M. Hosseini and S. V. Hum, "A semianalytical approach to designing high-transparency low-profile circular polarizers," IEEE Trans. Antennas Propag., vol. 66, no. 12, pp. 7138-7147, Dec. 2018.

[16] A. E. Karbowiak, "Theory of imperfect waveguides: the effect of wall impedance," Proceedings of the IEE - Part B: Radio and Electronic Engineering, vol. 102, no. 5, pp. 698-708, Sept. 1955.

[17] R. Elliott, "On the theory of corrugated plane surfaces," IRE Tran. Antennas Propag., vol. 2, no. 2, pp. 71-81, Apr 1954.

[18] R. Dybdal, "Waveguide applications of impedance surface," Ph.D. dissertation, The Ohio State University, Columbus, Ohio, USA, 1968.

[19] M. N. M. Kehn, "Modal analysis of all-walls longitudinally corrugated rectangular waveguides using asymptotic corrugations boundary conditions," IEEE Trans. Microw. Theory Techn., vol. 61, no. 11, pp. 38213837, Nov 2013.

[20] D. M. Pozar, Microwave engineering. John Wiley \& Sons, 2012.

[21] _ "A relation between the active input impedance and the active element pattern of a phased array," IEEE Trans. Antennas Propag., vol. 51, no. 9, pp. 2486-2489, Sept 2003.

[22] N. Marcuvitz, Waveguide handbook, 3rd ed. McGraw-Hill, 1951.

[23] F. Foglia Manzillo, R. Sauleau, N. Capet, and M. Ettorre, "Mode matching analysis of an E-plane $90^{\circ}$ bend with a square step in parallel plate waveguide," IEEE Antennas Wireless Propag. Lett., vol. 16, pp. 2187 - 2190, 2017.

[24] A. Ishimaru, R. Coe, G. Miller, and W. Geren, "Finite periodic structure approach to large scanning array problems," IEEE Trans. Antennas Propag., vol. 33, no. 11, pp. 1213-1220, Nov. 1985. 